\title{
The content and composition of organic matter in bottom sediments of the Yenisei River near Mining-Chemical Combine (MCC) Rosatom
}

\author{
Bondareva L.G. \\ Federal Scientific Center of Hygiene named after F.F. Erisman, Mytischi, Russia \\ lydiabondareva@gmail.com
}

doi: 10.36291/HIT.2019.bondareva.106

More than 50 years Krasnoyarsk Mining-Chemical Combine (MCC) manufactures weapon plutonium in single-pass production reactors cooled with water of the Yenisei River. Water discharge from these reactors is the major source of radioactive contamination of the Yenisei River. Sediment composition influences the level of radioactivity to a high degree. The total concentration of measured radionuclides (excluding ${ }^{40} \mathrm{~K}$ ) increases as the sediment decreases in size and becomes less well sorted and as the textural distribution of the sediment becomes more skewed toward fine material. Organic matter has important influences on the fate of environmental pollution in water dam reservoirs. The aim of the studies was to assess content of organic matter fractions in Yenisei River's bottom sediments, and to determine their influence on the content of heavy metals and PAHs. In sediment samples, the content of C organic (Corg), C extracted (Cex), C humic acid (Cha), C fulvic acid (Cfa) and C non-hydrolyzed (Cnh), buffer capacity, and content of heavy metals and PAHs was analyzed. The formation of radioactive flood-land deposits is provided by rapid deposition of suspended material in stagnant zones during periodical flood. Humus compounds contribute significantly to accumulation of radionuclides in the flood-land deposits and bottom sediments.

Radionuclides can also form stable, insoluble complexes with humic materials that tend to reduce radionuclide mobility. These insoluble complexes may be radionuclidehumate colloids that subsequently precipitate from solution or complexes of radionuclides and humic substances that sorb to clay minerals or other soil particulates strongly enough to immobilize the radionuclides. Colloid formation appears to be favored by increased radionuclide concentration and lowered $\mathrm{pH}$; however, the conditions that favor formation of insoluble complexes that sorb to particulates are still poorly understood.

Radiochemical analysis of separate fractions showed that about $20 \%$ of $\mathrm{Pu}$ and $\mathrm{Am}$ are associated with the organic fraction: $\mathrm{Pu}$ is nearly equally distributed between Cha and Cfa fractions, whereas Am is preferentially associated with the Cfa fraction (the most mobile fraction of humus matter). It was demonstrated in model experiments that the calciumhydrocarbonate type of water of the Yenisei River causes suppression of formation of mobile fulvate complexes of hydrolyzable radionuclides and, therefore, their transfer into the aqueous phase. In combination with the observed very high distribution coefficients of the radionuclides and low content of their mobile geochemical forms in flood-land deposits of the Yenisei River this suggest that they cannot contribute somewhat significantly to the secondary radioactive contamination of the river water by all mechanisms except migration by mechanical transfer. 